\title{
Riemann Hypothesis and Value Distribution Theory
}

\author{
Jinhua Fei \\ Chang Ling Company of Electronic Technology, Baoji, China \\ Email: feijinhuayoujian@msn.com
}

How to cite this paper: Fei, C.L. (2017) Riemann Hypothesis and Value Distribution Theory. Journal of Applied Mathematics and Physics, 5, 734-740. https://doi.org/10.4236/jamp.2017.53062

Received: March 4, 2017

Accepted: March 28, 2017

Published: March 31, 2017

Copyright (c) 2017 by author(s) and Scientific Research Publishing Inc. This work is licensed under the Creative Commons Attribution International License (CC BY 4.0).

http://creativecommons.org/licenses/by/4.0/

\begin{abstract}
Riemann Hypothesis was posed by Riemann in early 50's of the 19th century in his thesis titled "The Number of Primes less than a Given Number". It is one of the unsolved "Supper" problems of mathematics. The Riemann Hypothesis is closely related to the well-known Prime Number Theorem. The Riemann Hypothesis states that all the nontrivial zeros of the zeta-function lie on the "critical line" $\left\{s: \operatorname{Re} s=\frac{1}{2}\right\}$. In this paper, we use Nevanlinna's Second Main Theorem in the value distribution theory, refute the Riemann Hypothesis. In reference [7], we have already given a proof of refute the Riemann Hypothesis. In this paper, we gave out the second proof, please read the reference.
\end{abstract}

\section{Keywords}

Value Distribution Theory, Nevanlinna's Second Main Theorems, Riemann Hypothesis

\section{Introduction}

In the 19th century, the famous mathematician E. Picard obtained the pathbreaking result: Any non-constant entire function $f(z)$ must take every finite complex value infinitely many times, with at most one exception. Later, E. Borel, by introducing the concept of the order of an entire function, gave the above result a more precise formulation.

This result, generally known as the Picard-Borel theorem, lays the foundation for the theory of value distribution and since then it has been the source of many research papers on this subject. R. Nevanlinna made the decisive contribution to the development of the theory of value distribution. The Picard-Borel Theorem is a direct consequence of Nevanlinna theory.

In this paper, we use Nevanlinna's Second Main Theorem in the value distribu- 
tion theory; we got an important the conclusion by Riemann hypothesis. This conclusion contradicts the References [5] theorem 8.12 of the page 204, therefore we prove that Riemann hypothesis is incorrect.

\section{Some Results in the Theory of Value Distribution}

We give some notations, definitions and theorems in the theory of value distribution, its contents are in the references [1] and [6].

We write

$$
\log ^{+} x= \begin{cases}\log x & 1 \leq x \\ 0 & 0 \leq x<1\end{cases}
$$

It is easy to see that $\log x \leq \log ^{+} x$.

Let $f(z)$ be a non-constant meromorphic function in the circle $|z|<R, 0<R<+\infty$. we denote by $n(r, f)$ the number of poles of $f(z)$ on $|z| \leq r(0<r<R)$, each pole being counted with its proper multiplicity. Denote by $n(0, f)$ the multiplicity of the pole of $f(z)$ at the origin. For arbitrary complex number $a \neq \infty$, we denote by $n\left(r, \frac{1}{f-a}\right)$ the number of zeros of $f(z)-a$ on $|z| \leq r(0<r<R)$, each zero being counted with its proper multiplicity. Denote by $n\left(0, \frac{1}{f-a}\right)$ the multiplicity of the zero of $f(z)-a$ at the origin.

We write

$$
\begin{gathered}
m(r, f)=\frac{1}{2 \pi} \int_{0}^{2 \pi} \log ^{+}\left|f\left(r \mathrm{e}^{i \varphi}\right)\right| \mathrm{d} \varphi \\
N(r, f)=\int_{0}^{r} \frac{n(t, f)-n(0, f)}{t} \mathrm{~d} t+n(0, f) \log r .
\end{gathered}
$$

When $a \neq \infty$,

$$
N\left(r, \frac{1}{f-a}\right)=\int_{0}^{r} \frac{n\left(t, \frac{1}{f-a}\right)-n\left(0, \frac{1}{f-a}\right)}{t} \mathrm{~d} t+n\left(0, \frac{1}{f-a}\right) \log r
$$

and $T(r, f)=m(r, f)+N(r, f), T(r, f)$ is called the characteristic function of $f(z)$.

Lemma 2.1. If $f(z)$ is a analytical function in the circle $|z|<R(0<R<\infty)$. we have

$$
T(r, f) \leq \log ^{+} M(r, f) \leq \frac{\rho+r}{\rho-r} T(\rho, f) \quad(0<r<\rho<R)
$$

where $M(r, f)=\max _{|z|=r}|f(z)|$

Lemma 2.1 follows from the References [1], page 7.

Lemma 2.2. Let $f(z)$ be a non-constant meromorphic function in the circle $|z|<R(0<R<\infty) . \quad a_{\lambda}(\lambda=1,2, \cdots, h)$ and $b_{\mu}(\mu=1,2, \cdots, k)$ are the zeros and poles of $f(\mathrm{z})$ in the circle $|z|<\rho(0<\rho<R)$ respectively, each zero or pole appears as its multiplicity indicates, and $z=0$ is neither zero nor pole of the 
function $f(z)$, then, in the circle $|z|<\rho$, we have the following formula

$$
\log |f(0)|=\frac{1}{2 \pi} \int_{0}^{2 \pi} \log \left|f\left(\rho \mathrm{e}^{i \varphi}\right)\right| \mathrm{d} \varphi-\sum_{\lambda=1}^{h} \log \frac{\rho}{\left|a_{\lambda}\right|}+\sum_{\mu=1}^{k} \log \frac{\rho}{\left|b_{\mu}\right|} .
$$

This formula is called Jensen formula.

Lemma 2.2 follows from the References [1], page 3.

Lemma 2.3. Let $f(z)$ be the meromorphic function in the circle $|z| \leq R$, and $f(0) \neq 0, \infty, 1, \quad f^{\prime}(0) \neq 0$. when $0<r<R$, we have

$$
\begin{aligned}
T(r, f)< & 2\left\{N\left(R, \frac{1}{f}\right)+N\left(R, \frac{1}{f-1}\right)+N(R, f)\right\}+4 \log ^{+}|f(0)| \\
& +2 \log ^{+} \frac{1}{R\left|f^{\prime}(0)\right|}+36 \log \frac{R}{R-r}+5220
\end{aligned}
$$

This is a form of Nevanlinna's Second Main Theorem.

Lemma 2.3 follows from the References [1], theorem 2.4 of page 55 .

Lemma 2.4. Let $f(x)$ be decreasing and non-negative for $x \geq a$. Then the limit

$$
\lim _{N \rightarrow \infty}\left(\sum_{n=a}^{N} f(n)-\int_{a}^{N} f(x) \mathrm{d} x\right)=\eta
$$

exists, and that $0 \leq \eta \leq f(a)$. Moreover, if $f(x) \rightarrow 0$ as $x \rightarrow \infty$, then for $\xi \geq a+1$, we have

$$
\left|\sum_{a \leq n \leq \xi} f(n)-\int_{a}^{\xi} f(x) \mathrm{d} x-\eta\right| \leq f(\xi-1)
$$

The lemma 2.4 follows from the References [2], the theorem 8.2 of page 87 .

Lemma 2.5. When $\sigma \geq \frac{1}{2},|t| \geq 2$, we have

$$
|\zeta(\sigma+i t)| \leq c_{1}|t|^{\frac{1}{2}}
$$

Where $\zeta(s)$ is Riemann zeta function.

Lemma 2.5 follows from the References [3], the lemma 8.4 of page 188 .

Lemma 2.6. Let $f(z)$ be the analytic function in the circle $|z| \leq R$, let $M(r)$ and $A(r)$ denote the maxima of $|f(z)|$ and $\operatorname{Re} f(z)$ on $|z|=r$ respectively. Then for $0<r<R$, we have

$$
M(r) \leq \frac{2 r}{R-r} A(R)+\frac{R+r}{R-r}|f(0)|
$$

where $\operatorname{Re} s$ is the real part of the complex number s.

Lemma 2.6 follows from the References [4], page 175.

\section{Preparatory Work}

Let $s=\sigma+i t$ is the complex number, when $\sigma>1$, Riemann zeta function is

$$
\zeta(s)=\sum_{n=1}^{\infty} \frac{1}{n^{s}}
$$

When $\sigma>1$, we have 


$$
\log \zeta(s)=\sum_{n=2}^{\infty} \frac{\Lambda(n)}{n^{s} \log n}
$$

where $\Lambda(n)$ is Mangoldt function.

Lemma 3.1. If $t$ is any real number, we have

1) $0.0426 \leq|\log \zeta(4+i t)| \leq 0.0824$

2) $|\zeta(4+i t)-1| \geq 0.0426$

3) $0.917 \leq|\zeta(4+i t)| \leq 1.0824$

4) $\left|\zeta^{\prime}(4+i t)\right| \geq 0.012$

\section{Proof.}

1)

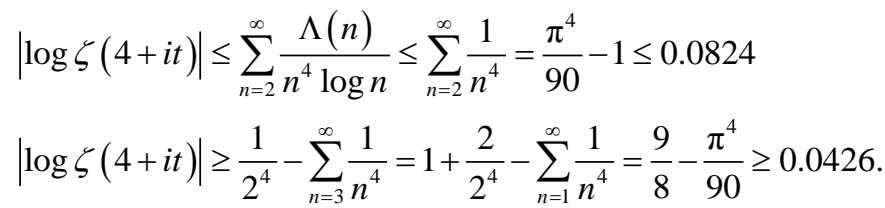

2)

$|\zeta(4+i t)-1|=\left|\sum_{n=2}^{\infty} \frac{1}{n^{4+i t}}\right| \geq \frac{1}{2^{4}}-\sum_{n=3}^{\infty} \frac{1}{n^{4}}$ $=1+\frac{2}{2^{4}}-\sum_{n=1}^{\infty} \frac{1}{n^{4}}=\frac{9}{8}-\frac{\pi^{4}}{90} \geq 0.0426$.

3)

$$
\begin{aligned}
& |\zeta(4+i t)|=\left|\sum_{n=1}^{\infty} \frac{1}{n^{4+i t}}\right| \leq \sum_{n=1}^{\infty} \frac{1}{n^{4}}=\frac{\pi^{4}}{90} \leq 1.0824 \\
& |\zeta(4+i t)|=\left|\sum_{n=1}^{\infty} \frac{1}{n^{4+i t}}\right| \geq 1-\sum_{n=2}^{\infty} \frac{1}{n^{4}}=2-\sum_{n=1}^{\infty} \frac{1}{n^{4}}=2-\frac{\pi^{4}}{90} \geq 0.917 .
\end{aligned}
$$

4) $\left|\zeta^{\prime}(4+i t)\right|=\left|\sum_{n=2}^{\infty} \frac{\log n}{n^{4+i t}}\right| \geq \frac{\log 2}{2^{4}}-\sum_{n=3}^{\infty} \frac{\log n}{n^{4}}$.

by Lemma 2.4 , we have

$$
\sum_{n=3}^{\infty} \frac{\log n}{n^{4}}=\int_{3}^{\infty} \frac{\log x}{x^{4}} \mathrm{~d} x+\eta
$$

where $0 \leq \eta \leq \frac{\log 3}{3^{4}}$

$$
\int_{3}^{\infty} \frac{\log x}{x^{4}} \mathrm{~d} x=-\frac{1}{3} \int_{3}^{\infty} \log x \mathrm{~d} x^{-3}=\frac{\log 3}{3^{4}}+\frac{1}{3} \int_{3}^{\infty} x^{-4} \mathrm{~d} x=\frac{\log 3}{3^{4}}-\frac{1}{3^{2}} \int_{3}^{\infty} \mathrm{d} x^{-3}=\frac{\log 3}{3^{4}}+\frac{1}{3^{5}} .
$$

Therefore

$$
\begin{aligned}
& \sum_{n=3}^{\infty} \frac{\log n}{n^{4}} \leq \frac{\log 3}{3^{4}}+\frac{1}{3^{5}}+\frac{\log 3}{3^{4}} \\
& \left|\zeta^{\prime}(4+i t)\right| \geq \frac{\log 2}{2^{4}}-\frac{2 \log 3}{3^{4}}-\frac{1}{3^{5}} \geq 0.012 .
\end{aligned}
$$

This completes the proof of Lemma 3.1.

Now, we assume that Riemann hypothesis is correct, and abbreviation as RH. In other words, when $\sigma>\frac{1}{2}$, the function $\zeta(\sigma+i t)$ has no zeros. The function 
$\log \zeta(\sigma+i t)$ is a multi-valued analytic function in the region $\sigma>\frac{1}{2}, t \geq 1$. we choose the principal branch of the function $\log \zeta(\sigma+i t)$, therefore, if $\zeta(\sigma+i t)=1$, then $\log \zeta(\sigma+i t)=0$.

Let $\delta=\frac{1}{100}, \quad c_{1}, c_{2}, \cdots$, is the positive constant.

Lemma 3.2. If RH is correct, when $\delta=\frac{1}{100}, \sigma \geq \frac{1}{2}+2 \delta, t \geq 16$, we have

$$
|\log \zeta(\sigma+i t)| \leq c_{2} \log t+c_{3}
$$

Proof. In Lemma 2.6, we choose $f(z)=\log \zeta(z+4+i t)$, $R=\frac{7}{2}-\delta, r=\frac{7}{2}-2 \delta, t \geq 16$.

Because $\log \zeta(z+4+i t)$ is the analytic function in the circle $|z| \leq R$, by Lemma 2.6, in the circle $|z| \leq r$, we have

$$
|\log \zeta(z+4+i t)| \leq \frac{7}{\delta}(A(R)+|\log \zeta(4+i t)|)
$$

by Lemma 2.5 , we have

$$
A(R)=\max _{\left|z-z_{0}\right|=R} \log |\zeta(z+4+i t)| \leq \frac{1}{2} \log t+\log c_{1}
$$

by Lemma 3.1, we have

$$
|\log \zeta(z+4+i t)| \leq c_{2} \log t+c_{3}
$$

therefore, when $\sigma \geq \frac{1}{2}+2 \delta$, we have

$$
|\log \zeta(\sigma+i t)| \leq c_{2} \log t+c_{3}
$$

This completes the proof of Lemma 3.2.

Lemma 3.3. If $\mathrm{RH}$ is correct, when $\delta=\frac{1}{100}, t \geq 16, \rho=\frac{7}{2}-2 \delta$, in the circle $|z| \leq \rho$, we have

$$
N\left(\rho, \frac{1}{\zeta(z+4+i t)-1}\right) \leq \log \log t+c_{4}
$$

Proof. In the Lemma 2.2, we choose

$f(z)=\log \zeta(z+4+i t), R=\frac{7}{2}-\delta, \rho=\frac{7}{2}-2 \delta . \quad a_{\lambda}(\lambda=1,2, \cdots, h)$ are the zeros of the function $\log \zeta(z+4+i t)$ in the circle $|z|<\rho$, each zero appears as its multiplicity indicates. Because the function $\log \zeta(z+4+i t)$ has no poles in the circle $|z|<\rho$, and $\log \zeta(4+i t)$ is not equal to zero, we have

$$
\log |\log \zeta(4+i t)|=\frac{1}{2 \pi} \int_{0}^{2 \pi} \log \left|\log \zeta\left(4+i t+\rho \mathrm{e}^{i \varphi}\right)\right| \mathrm{d} \varphi-\sum_{\lambda=1}^{h} \log \frac{\rho}{\left|a_{\lambda}\right|}
$$

by Lemma 3.1 and Lemma 3.2, we have

$$
\sum_{\lambda=1}^{h} \log \frac{\rho}{\left|a_{\lambda}\right|} \leq \log \log t+c_{4} .
$$


Because $z=0$ is neither zero nor pole of the function $\log \zeta(z+4+i t)$, we have

$$
\begin{aligned}
\sum_{\lambda=1}^{h} \log \frac{\rho}{\left|a_{\lambda}\right|} & =\int_{0}^{\rho}\left(\log \frac{\rho}{t}\right) \mathrm{d} n\left(t, \frac{1}{f}\right)=\left[\left(\log \frac{\rho}{t}\right) n\left(t, \frac{1}{f}\right)\right]_{0}^{\rho}+\int_{0}^{\rho} \frac{n\left(t, \frac{1}{f}\right)}{t} \mathrm{~d} t \\
& =\int_{0}^{\rho} \frac{n\left(t, \frac{1}{f}\right)}{t} \mathrm{~d} t=N\left(\rho, \frac{1}{f}\right) \\
& =N\left(\rho, \frac{1}{\log \zeta(z+4+i t)}\right) \geq N\left(\rho, \frac{1}{\zeta(z+4+i t)-1}\right) .
\end{aligned}
$$

This completes the proof of Lemma 3.3.

\section{Proof of Conclusion}

Theorem. If RH is correct, when $\sigma \geq \frac{1}{2}+4 \delta, \delta=\frac{1}{100}, t \geq 16$, we have

$$
|\zeta(\sigma+i t)| \leq c_{8}(\log t)^{c_{6}}
$$

Proof. In Lemma 2.3, we choose $f(z)=\zeta(z+4+i t)$, $t \geq 16, R=\frac{7}{2}-2 \delta, r=\frac{7}{2}-3 \delta$. by Lemma3.1, we have $f(0)=\zeta(4+i t) \neq 0, \infty, 1$, and $\left|f^{\prime}(0)\right|=\left|\zeta^{\prime}(4+i t)\right| \geq 0.012$, $|f(0)|=|\zeta(4+i t)| \leq 1.0824$. Because $\zeta(z+4+i t)$ is the analytic function, and it have neither zeros nor poles in the circle $|z| \leq R$, we have

$$
N\left(R, \frac{1}{f}\right)=0, \quad N(R, f)=0
$$

therefore, by Lemma 3.3, we have

$$
T(r, \zeta(z+4+i t)) \leq 2 \log \log t+c_{5}
$$

In Lemma 2.1, we choose $R=\frac{7}{2}-2 \delta, \rho=\frac{7}{2}-3 \delta, r=\frac{7}{2}-4 \delta$. by the maximal principle, in the circle $|z| \leq r$, we have

$$
\log ^{+}|\zeta(z+4+i t)| \leq c_{6} \log \log t+c_{7}
$$

Therefore, when $\sigma \geq \frac{1}{2}+4 \delta$, we have

$$
\begin{gathered}
\log ^{+}|\zeta(\sigma+i t)| \leq c_{6} \log \log t+c_{7} \\
\log |\zeta(\sigma+i t)| \leq c_{6} \log \log t+c_{7} \\
|\zeta(\sigma+i t)| \leq c_{8}(\log t)^{c_{6}}
\end{gathered}
$$

This completes the proof of Theorem.

The conclusion of Theorem contradicts the References [5] theorem 8.12 of the page 204, therefore we prove that Riemann hypothesis is incorrect.

\section{References}

[1] Zhang, G.H. (1993) Theory of Entire and Meromorphic Functions. American Ma- 
thematical Society.

[2] Hua, L.K. (1982) Introduction to Number Theory. Springer-Verlag, Berlin.

[3] Bateman, P.T. and Harold, G. (2004) Diamond. Analytic Number Theory, World Scientific Publishing.

[4] Titchmarsh, E.C. (1939) The Theory of Functions. Oxford University Press, Qxford.

[5] Titchmarsh, E.C. (1986) The Theory of the Riemann Zeta Functions. Oxford University Press, Oxford.

[6] Yang, L. (1993) Value Distribution Theory. Springer, Berlin.

[7] Jin, H.F. (2016) About the Riemann Hypothesis. Journal of Applied Mathematics and Physics, 4, 561-570.

Submit or recommend next manuscript to SCIRP and we will provide best service for you:

Accepting pre-submission inquiries through Email, Facebook, LinkedIn, Twitter, etc. A wide selection of journals (inclusive of 9 subjects, more than 200 journals)

Providing 24-hour high-quality service

User-friendly online submission system

Fair and swift peer-review system

Efficient typesetting and proofreading procedure

Display of the result of downloads and visits, as well as the number of cited articles

Maximum dissemination of your research work

Submit your manuscript at: http://papersubmission.scirp.org/

Or contact jamp@scirp.org 